\title{
MUSIKINSTRUMENTE UND MUSIKAUSÜBUNG IN DER EISENZEIT MITTELEUROPAS - ANHAND VON BILDQUELLEN UND ORIGINALFUNDEN
}

\author{
A LEX A N DRINE EIBNER - CLEMENS EIBNER
}

\begin{abstract}
Musical Instruments and Music Practice During the Iron Age in Central Europe - Based on Pictorial Sources and Original Finds. Besides the already traditionally widely treated Iron Age music instruments, which slightly overstep the field of Situla Art, special attention is paid here to the musicians. In this paper the string and wind instruments known from the East Hallstatt region as well as Situla Art and forms of lyres from West Hallstatt region will be presented. Additionally, small figurines and original finds provide evidence of auloi, syringes made of various materials and carnices (these, however, from the La Tène period). Bells and ideophones are also attested in West Hallstatt region. Special attention is paid to the music practice, social status as well as the gender role of music players. Besides singing, many dance forms are attested; especially in East Hallstatt region the female musicians and dancers must be emphasized.
\end{abstract}

Keywords: Central Europe, Iron Age, Hallstatt culture, musical instruments, social status, role of women, musical and dancing performance.

\section{EINLEITUNG}

Musikinstrumente der Eisenzeit (Eibner A. 1986; 1994; Pomberger 2016) sind im Osthallstattkreis in Darstellungen vorwiegend auf Keramikgefäßen und im Bereich der Situlenkunst auf Bronzegefäßen auf uns gekommen. Das Gebiet des Osthallstattkreises umfasst den Ostalpenrand bis in den Pannonischen Raum, während die Werke der Situlenkunst von Alttirol ${ }^{1}$ über Istrien, Kroatien bis ins ehemalige Kronland Krain verbreitet sind sowie von Kärnten bis Salzburg und Oberösterreich.

Als Originalfunde werden hier nicht nur die erhaltenen Fragmente von Instrumenten ${ }^{2}$ angesprochen, sondern auch die Statuetten mit ihren Details.

\section{DARGESTELLTE MUSIKINSTRUMENTE - BILDBELEGE}

\section{Saiten- und Blasinstrumente im Osthallstattkreis}

Im Osthallstattkreis finden sich Saiten- und Blasinstrumente.

1. Als Chordophone sind zwei Leierformen und die Harfe überliefert. Die phorminxartige Hallstattleier (Eibner A. 1994, 303 f.) ist in Ritztechnik von Sopron/ Tumulus 27 (Taf. I: 1) und von Reichersdorf/Grab 20
(Taf. IV: 3) überliefert sowie in fein eingestochenen Pünktchen von der Býčí skála-Höhle bei Brno (Taf. II: 3; Eibner A. 1999). In Punkt-Buckel-Technik findet sie sich auf der Ziste XII von Kleinklein-Kröllkogel (Egg/Munir 2013, 215-217, Beilage 22: 2) und auf der Situla Typus Kurd von Sesto Calende/Kriegergrab A (Taf. I: 2; Eibner A. 1981, Abb. 13).

Die Rahmenleier vom Typ Sopron (Eibner A. 1994, 304 f.; Lochner 2011, 10-12) ist in Ritztechnik von Sopron/Tumulus 28 (Taf. I: 4) und dem Unbekannten Tumulus - 96 oder 98 (Taf. IV: 1) überliefert, die weiteren von Nové Košariská und Dolné Janíky (Taf. IV: 2) sowie von Rabensburg (Dobiat 1982, 314, Anm. 52) und Leithaprodersdorf (Eibner A. 1986, mit f. n. 2) sind in Graphitmalerei erhalten, das Fragment von Ernstbrunn in Rollrädchentechnik (Dobiat 1982, 320 f., Abb. 26). Die Winkel- oder dreieckige Rahmenharfe von Nové Košariská (Eibner A. 1994, 306) ist ebenfalls in Graphitmalerei dargestellt.

2. Als Aerophon ist nur der Hallstattaulos überliefert. Der Hallstattaulos mit im rechten Winkel angesetztem, rinderhornförmigem Schalltrichter (Eibner A. 1994, 307 f.) findet sich als Doppelaulos in Punkt-Buckel-Technik auf der Bronzeziste XIII (Taf. II: 1) und dem zugehörigen Deckel von Kleinklein-Kröllkogel sowie als einfacher Aulos in feinen Pünktchen auf einem Bronzefragment von der Býčí skála-Höhle (Taf. II: 3).

\footnotetext{
1 Umfasst Tirol und Osttirol in Österreich sowie Alto Adige und Trentino (Südtirol) in Norditalien. Die geographische Umschreibung venetisch bzw. slowenisch haben wir als Begriff eingeführt, um das Gemeinsame der Darstellungen auf den jeweiligen Situlen besser ansprechen zu können.

2 Unter den überlieferten Instrumenten finden sich alle Gattungen nach E. M. Hornbostel und C. Sachs (1914).
} 


\section{Instrumentenformen in der Situlenkunst}

Im Bereich der Situlenkunst finden sich alle bekannten Instrumentenformen.

1. Als Chordophon ist nur die Situlenleier überliefert. Die Situlenleier (Eibner A. 1994, 304) hat vier, aber auch fünf Saiten ${ }^{3}$ (Taf. I: 5) und ein geschwungenes Joch, das in die Jocharme übergeht mit je einem Knopf an den Umbrüchen. Zumeist ist sie im zweiten Fries jener Situlen wiedergegeben, die das Situlenfest zeigen. ${ }^{4}$

2. Als Aerophone sind die Syrinx und das Horn überliefert. Die Syrinx/Panflöte (Eibner A. 1994, 306 f.; Pomberger 2016, 62 f., Abb. 20; 21) besteht aus vier (Taf. I: 5; II: 4) bis sieben abgeschrägten Röhren, die untereinander zum Teil in kunstvoller Verschnürung verbunden sind. Sie kommen im selben Kontext vor wie die Situlenleiern, wo man beim Fest gemeinsam aufspielt. Allerdings kennt man Syrinxbläser in diesem Zusammenhang auf slowenischen Situlen nur als Solisten (Eibner A. 2018a, Taf. 16: 31a, 31b).

Das Horn (Eibner A. 1994, 308-310; 2013), gekrümmt, zum Teil mit Hornfessel, findet sich in Szenen mit kriegerischem Hintergrund dargestellt wie in der Hand des Anführers von Infanteristen (Taf. III: 1) als auch von Kavalleristen ${ }^{5}$ (Taf. III: 7) sowie Signal gebend, um Hilfe herbeizurufen (Taf. III: 2; Eibner A. 2018b, 113 f., Abb. 7; 2013, 159 f., Taf. 2; 3). In estensischen Heiligtümern sind vollgerüstete Hornbläser auf Votivblechen wiedergegeben (Taf. III: 5, 6; Eibner A. 2018b, 113 f., Abb. 7: a, b). Wohl ein Numen stellt der behelmte, geflügelte Hornbläser mit den an ihm aufsteigenden Fabelwesen auf dem Bronzespiegel Arnoaldi von Bologna dar (Taf. III: 4; Eibner A. 2013, 160, Taf. III: 14). Felsbilder aus der Valcamonica zeigen ebenfalls Hörner, zum Teil mit Hornfessel (Taf. III: 3; Eibner A. 2013, 160, Taf. 2: 10a; 3: 15), in Händen von Kriegern oder in Zusammenhang mit Tänzern (Eibner A. 2012, 177-180, Taf. 6: 18-23; 7: 24-26 mit Springtänzern).

3. Als Ideophone sind ein Sistrum und Schellen überliefert. Ein Sistrum (?) in Treibtechnik in der Hand einer Frau mit hüftlangem Schleier auf einer
Bronzeblechscheibe dargestellt, stammt aus einem mit Votiven gefüllten Opferbrunnen von Musile di Piave. ${ }^{6}$

Schellen sind am Halsgurt von Pferden (Pomberger 2017, 20-23, Abb. 2; 3) auf der Situla Vače (Taf. III: 9) zu erkennen.

4. Als Membranophon könnte ein Felsbild angesprochen werden. Eine Trommel ist möglicherweise auf einem Felsbild aus der Valcamonica wiedergegeben (Eibner A. 2008, 181, Taf. 10: 34; Pomberger 2016, 55 f., Abb. 14: b).

\section{Leierformen im Westhallstattkreis}

Im Westhallstattkreis findet sich nur eine Leierform. Als Chordophon ist nur die Leier vom Typ Oberpfalz überliefert. Dieser Leiertyp (Eibner A. 1994, 306) findet sich in Rollrädchentechnik als Einzeldarstellung auf Gefäßen von Schirndorf in Oberbayern (Taf. I: 3).

\section{STATUETTEN UND ORIGINALFUNDE}

\section{Osthallstattkreis}

Im Osthallstattkreis finden sich Aerophone und ein Membranophon.

1. Als Aerophone sind der Hallstattaulos und die Carnyx sowie Knochenpfeifchen überliefert.

Ein Hallstattaulos ist durch die Bronzestatuette von Százhalombatta bei Budapest bekannt geworden (Taf. II: 2; Eibner A. 1999, Abb. 1; 2017, 75, Abb. 3: 1). Einen Carnyxbläser zeigt die nackte Bronzestatuette - vermutlich aus Kondoros in Kom. Békés (Taf. II: 10; Veres 2009). Die Knochenpfeifchen sind meist aus Vogelknochen hergestellt und finden sich vor allem im Bereich der Kalenderbergkultur (Pomberger 2016, 57 f., Abb. 15: a, b; 16: $\mathrm{a}-\mathrm{c}$ ).

2. Als Membranophon ist eine Keramiktrommel überliefert. In Becherform ist sie von Inzersdorf ob der Traisen bekannt geworden (Pomberger 2016, 55 f., Abb. 14).

\footnotetext{
3 Zehn Saiten sind auf den Situlen Certosa und Dürrnberg-Kranzbichl zu erkennen. Herr Dr. Wolfgang Lobisser denkt dabei an fünf Doppelsaiten, die man mit der linken Hand gut abgreifen könnte, was eigentlich fünf gleich gestimmte Saitenpaare wahrscheinlich macht. „Meine (Stege) sind zwar nicht vollständig erhalten, aber wenn ich sie spiegle, komm ich bei beiden auf zehn Saitenkerben", sodass man anhand der Abnutzungsspuren an den Stegen von doppelsaitiger Bespannung ausgehen kann. Für seine Expertise möchten wir uns an dieser Stelle sehr herzlich bei ihm bedanken.

4 Als musikalische Umrahmung des Festes ist das Leierspiel auf allen venetischen Situlen zu finden, ergänzt noch durch Syrinxbläser (Eibner A. 2018a, Taf. 16: 31).

5 Aus dem Reitia Heiligtum in Este, wegen der zwei Löcher im Pferdekörper wohl als Votiv verwendet (Eibner A. $2018 b$, 113 f., Abb. 7: c).

6 Wurde als Blüte gedeutet, könnte aber ein Sistrum sein (Tomedi 2001, 18 f., Abb. 5).
} 


\section{Situlenkunst}

Im Bereich der Situlenkunst finden sich Chordophone, Aerophone und Ideophone.

1. Als Chordophone sind Leier und Harfen überliefert. Die Leierfragmente aus Holz - Wirbelkörper- und zwei Stegfragmente - stammen von der Gewerbesiedlung im Ramsautal am Dürrnberg bei Hallein (Lobisser, im Druck).

Eine Winkelharfe wird von der Bronzestatuette eines nackten, auf einem Faltstuhl sitzenden Musikers von Sesto al Reghena (Prov. Pordenone) gespielt (Lochner 2011, 12-14, Abb. 10; 11; Tomedi 2001, 26, Abb. 13). Ein Winkelharfenfragment aus Rothirschgeweih stammt aus der Siedlung (Heiligtum?) Pirchboden ob Fritzens. Erhalten sind das Jochfragment mit sieben Bronzestiften sowie eine Inschrift im rätischen Alphabet auf der Jochunterseite (Tomedi 2001, 31 f., Abb. 19).

2. Als Aerophone sind Syrinx, Carnyx und Horn sowie Flöten überliefert. Die Syrinxbläser sind durch zwei Bronzestatuetten vertreten (Salzani 1976, Taf. 31: 2; Tomedi 2001, Abb. 9; 10), die eine ist nackt und spielt auf einer trapezförmigen Syrinx, die andere ist bekleidet und hält eine rechteckige Syrinx (Haas 1985, 22 f.) waagerecht vor der Brust. Syrinxfragmente aus Schafsknochen, ${ }^{7}$ Holunderröhren und durch Draht verbundene Bronzeröhren zeigen uns welch verschiedene Materialien man zur Syrinxherstellung verwendet hat (Pomberger 2016, 62 f., Abb. 20; 21; Sölder 2008, 224, Abb. 1). Carnyxfragmente werden in Depots niedergelegt wie in Sanzeno und in Leisach, ${ }^{8}$ hier fanden sich Teile von Rohren und die Ohren eines eberkopfförmigen Schalltrichters.

Ein Röhrenfragment mit Grifflöchern aus Holz von einer Flöte oder einem Aulos stammt aus der Gewerbesiedlung im Ramsautal am Dürrnberg bei Hallein (Lobisser, im Druck).

Als Hornbläser ist die Bronzestatuette von Idrija pri Bači anzusprechen (Taf. III: 8), obwohl Horn, Schild und Lanze fehlen, die sich jedoch rekonstruieren lassen (Eibner A. 2000, Abb. 1). Einen Hornisten stellt wohl die nackte Bronzestatuette mit einem nur schwach gekrümmten Horn in der Hand von Oberhofen-Pircherwald dar. Ihr Kopf mit dem nach oben gerichteten Blick passt wie auch der halb offene Mund zu einem Sänger (Pöll/Tomedi/Zanier 2019, bes. 97). Die Bronzestatuette aus dem Oppidum Hradiště bei Stradonice ist nackt und trägt das Horn mit Abschlussscheibe quasi geschultert (Eibner A. 2013, 160, Taf. 3: 3). Das Grünsteinrelief von Bormio am Stilfser Joch ist nur als Fragment erhalten. Es zeigt in der Bildmitte ein großes Feldzeichen und eine Lanze mit angehängtem Rundschild, denen sich von links ein Hornbläser nähert, während auf der anderen Seite ein größerer, frontal wiedergegebener Mann mit Hörnerhelm und Körper deckendem Tierfellschild steht, der das Feldzeichen in der weggestreckten rechten Hand hält - eine männliche Gottheit (?; Eibner A. 2008, 185, Anm. 106; 2013, 160, Taf. 3: 11; 2018b, 110-114, Abb. 6; 7). Die Szene wird von M. Egg $(2012,104)$ als Waffenweihung gedeutet.

Ein Rinderhorn aus der linken Hornscheide eines Hausrindes, wahrscheinlich als Signalhorn genutzt, fand sich im Salzbergwerk von Hallstatt (Tomedi 2001, 27 f., Abb. 14).

Die Flöten - Kerb- und Kernspaltflöten - verwendet als Signalflöten, sind meist aus Vogelknochen hergestellt. Es finden sich aber auch Gefäßflöten (Pomberger 2016, 58 f., Abb. 17; Tomedi 2001, 15 f., Abb. 4).

3. Als Ideophone sind Klappern, Rasselstäbe, Gefäßrasseln, Bommeln und Schellen überliefert. Ein Tintinnabulum zeigt in Treibtechnik auf beiden Seiten thronende Frauen mit Wollarbeiten sowie mit Spinnen und Weben beschäftigt (Eibner A. 2018a, 100-102, Taf. 15: 30b). Die Rasselstäbe sind mit verziertem Bronzeblech verkleidet, daran hängen an feinen Bronzekettchen montiert Klapperbleche bzw. kleine Pyxiden (Laharnar/Turk 2018, 75-79, Abb. 85). Die tönernen Gefäßrasseln ${ }^{9}$ haben häufig Vogelgestalt, sind aber auch birnförmig oder in Form eines Gefäßes und als Klapperpuppen gestaltet, mit kleinen Tonkügelchen bzw. Steinchen im Gefäßinneren. Geschlitzte Bronzebommeln sind rund, oval oder doppelkonisch, mit oder ohne Fuß und zieren z. B. einen Bronzegürtel aus der Býćí skála-Höhle (Pomberger 2017, 17-20, Abb. 1). Bronzeschellen sind geschlitzt, z. B. als Rasselkörper in einer Pferdetrense eingehängt (Pomberger 2017, 20-23, Abb. 2).

\section{Westhallstattkreis}

Im Westhallstattkreis sind vor allem Ideophone $\mathrm{zu}$ finden. Als Ideophone sind ein Sistrum und Schellen überliefert. Ein Sistrum aus Bronze ist von Hochborn (Kr. Alzey-Worms) bekannt (Tomedi 2001, 18 f., Abb. 6). Geschlitzte Bronzeschellen - ku-

\footnotetext{
7 Die Tone $\mathrm{c}^{3}-\mathrm{g}^{4}$ passen gut zu den in antiken Schriftquellen überlieferten hohen Tönen. Überliefert ist ebenfalls, dass Syrinxbläser im Zuge der Aufmärsche zur Begleitung des Schautanzes mitwirkten (Tomedi 2001, 21-23, Abb. 7; 8).

8 Auf dem Burgstall in Leisach bei Lienz innerhalb eines Heiligtums gefunden, wobei P. Gleirscher $(2014,113,115,117$, Abb. 13: 3$)$ auf Ähnlichkeiten mit Eberstandarten hinweist.

9 Zu finden meist in Kinder- und Frauengräbern (Pomberger 2016, 46-51, Abb. 1-6; 2017, 16).
} 
gelförmig, oval oder doppelkonisch - finden sich vorwiegend in der Hallstattkultur (Pomberger 2017, 20-23, bes. Abb. 3: 2). Tulpenförmige zwischen zwei in Bronze gefassten Eberhauern montierte Schellen sind als Pferdebrustschmuck im Tumulus 4 vom Bettelbühl zu Tage gekommen (Krausse u. a. 2017, 115 f., Abb. 9). ${ }^{10}$

\section{Weitere Statuen und Originalinstrumente}

Weitere Statuen und Originalinstrumente sind im Bereich von Frankreich bis zum Donauknie in Ungarn überliefert. Die Steinstatue aus Saint-Symphorien-en-Paule (Dép. Côtes-d'Armor) ${ }^{11}$ hält das phorminxartige Instrument mit acht Saiten frontal vor dem Oberkörper. Die Carnyx (Hunter 2019) besteht aus einem hoch aufgebogenen Rohr mit tierkopfförmigem Schalltrichter (Eibner A. 2013, 161, Taf. 5: 20) bzw. mit verzierter Abschlussscheibe (Préhistoire 2002, 112-114, Abb. 79-82). Im Heiligtum von Tintignac à Naves (Dép. Corréze) haben sich in einem Waffendepot mindestens sieben Schalltrichter (Taf. II: 6-9) erhalten, darunter ein schlangenkopfförmiger ${ }^{12}$ (Taf. II: 8). Carnyxbläser sind am Gundestrupkessel auf der Innenplatte mit "Kriegerzug" dargestellt (Taf. II: 5; Eibner A. 2013, Taf. 5: 18), während sich ein nackter Hornbläser als nicht ausgeführte Vorzeichnung auf der Rückseite der Innenplatte mit "Radgott" eingraviert findet - mit einem gekrümmten, zum Mund geführten Horn, das in einen Tierkopf mit weit aufgerissenem Maul endet (Eibner A. 2013, 162, Taf. 4: 16).

\section{SÄNGER}

Sänger sind aus beiden Bereichen bekannt. Als Darstellung aus dem Osthallstattkreis in fein eingestochenen Pünktchen auf einem Fragment aus der Býčí skála-Höhle zu finden (Taf. II: 3). Hier lässt sich ein Mann mit zurückgebogenem Kopf und nach oben gerichtetem Blick erkennen - zu rekonstruieren als Sänger, der sich mit der Leier begleitet. ${ }^{13}$ Als nackte Bronzestatuette ist aus dem Situlenbereich mit gleicher Kopfhaltung und geöffnetem Mund ein Sänger vom Pircherwald in Oberhofen erhalten (Pöll/Tomedi/Zanier 2019, 97-100, Abb. 5; 6).

\section{MUSIZIERPRAXIS - SOLISTEN UND ENSEMBLEMUSIKER}

\section{Osthallstattkreis}

Mit Musizierpraxis im Osthallstattkreis beschäftigte sich A. Eibner (2011, 29-34). Als Solisten zu werten sind der Musiker von Sopron/Tumulus 27 mit seiner Hallstattleier, der vor drei Frauen, den Schicksalsgöttinnen musiziert (Taf. I: 1; Eibner C. 2012) und ein weiterer von Reichersdorf, der zum Tanz aufspielt ${ }^{14}$ (Taf. IV: 3). Mit Y-förmigem Körper gehen weitere Musiker mit einer Rahmenleier vom Typ Sopron auf Frauen mit erhobenen Händen $\mathrm{zu}$, darunter finden sich auch Leierspielerinnen (Taf. IV: 2). Zwei Harfenspielerinnen sind von Nové Košariská/Tumulus IV bekannt (Pomberger 2016, 65-67, Abb. 23: a) und ein ithyphallischer Aulosbläser findet sich mit seinem einfachen Rohrblattinstrument auf dem Fragment von der Býćí skála-Höhle ${ }^{15}$ (Taf. II: 3).

Als Ensemblemusiker spielen sie zu zweit auf wie die Männer in Hosen. ${ }^{16}$ Diese stehen sich mit der Rahmenleier gegenüber und spielen zum Tanz auf (Taf. I: 4) oder gehen hintereinander her wie die Musikerin hinter einem solchen Musiker in Hosen (Taf. IV: 1) sowie die beiden Musiker mit der Hallstattleier auf der Bronzeziste XII vom Kröllkogel (Egg/Munir 2013, 215-217, Beilage 22: 2), die in der Natur aufzuspielen scheinen. Aulosbläser mit einem Hallstattaulos musizieren zueinander gedreht auf der Ziste XIII vom Kröllkogel ${ }^{17}$ (Taf. II: 1) sowie

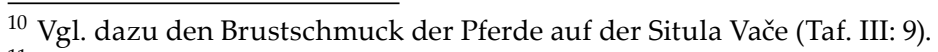

${ }^{11}$ In einer befestigten Hofanlage mit drei weiteren Statuen ausgegraben (Préhistoire 2002, 119-123, Abb. 87; Rieckhoff 2002, 129 f., Kat.-Nr. 1-4).

${ }^{12}$ Neben eberkopfförmigen (Taf. II: 6, 7, 9) sind noch rinder- und wolfskopfförmige bekannt (Eibner A. 2013, 161 f., Taf. 5).

${ }^{13}$ Der zurückgebogene Kopf ist die typische Haltung eines Sängers (Eibner A. 1999, Abb. 3; 2018a, 109). Im antiken Griechenland war die Kithara Begleitinstrument für epische Gesänge, das wird auch von den Barden überliefert, wofür sich Hinweise in zusammengesetzten Personennamen finden (Préhistoire 2002, 122 f.; Rieckhoff 2002, 130; Tomedi 2001, 23-26, Abb. 13).

${ }^{14}$ Eingeritzt als einzelne Figuren auf den Leerflächen zwischen den auf den Bauch herabhängenden Dreiecken - ein Leierspieler zwischen zwei Frauenpaaren.

${ }^{15}$ Vom Leierspieler ist nur der Oberkörper mit zurückgebogenem Kopf erhalten und von der Leier nur mehr der Ansatz des Jocharmes mit dem Querjoch (Eibner A. 1999, 46 mit Abb. 3 als Rekonstruktion).

${ }^{16}$ Hosen sind hier zum ersten Mal innerhalb der Kalenderbergkultur dargestellt (Taf. I: 4; IV: 1), zu finden auf Gefäßen aus der Nekropole von Sopron (Eibner A. 2011, 30, Abb. 30; 32).

${ }^{17}$ Zum Teil sind die Figuren isoliert unter Tieren eingestreut, erst mit der Ziste XIII ist ein lesbares Geschehen dargestellt (Egg 2013, 462, 470-472; Eibner A. 2017, 73-75, Abb. 2).
} 
auf dem zugehörigen Deckel und fungieren dabei gleichzeitig als Trenner zwischen den Bildszenen (Egg/Munir 2013, Taf. 39: 2; Beilage 23).

\section{Situlenkunst}

Mit Musizierpraxis im Bereich der Situlenkunst beschäftigte sich A. Eibner (2011, 34-38). Als Solist findet sich nur der Syrinxbläser unter den Festteilnehmern sitzend auf den slowenischen Situlen Magdalenska gora-Preloge (Tumulus XIII/Grab 55) und Vače.

Als Ensemble sitzen hingegen Leierspieler und Syrinxbläser auf den venetischen Situlen um den Trankbehälter herum und spielen beim Fest auf. Nur beim Tanz und beim musischen Agon spielt man auf zwei gleichartigen Instrumenten wie die beiden behelmten Musiker mit Situlenleiern beim Springtanz (Taf. IV: 5) und die zwei Syrinxbläser beim Wettstreit auf der Situla Providence.

Als Hornbläser sind sie, wie beschrieben, nur im Zusammenhang mit dem Kriegswesen oder auf Votiven dargestellt (Taf. III: 1-2, 5-7).

\section{ZUM SOZIALSTATUS UND ZUR GESCHLECHTERROLLE DER MUSIKER}

\section{Situlenkunst}

Der Sozialstatus der Musiker lässt sich nur im Bereich der Situlenkunst erkennen:

\section{1. anhand des Helmes:}

Musiker mit langem Gewand erweisen sich dadurch als Angehörige des Kriegerstandes, zu deren Ausbildung jedoch nicht nur der Kampf, sondern auch Musik und Tanz gehörten (Erker 2018). Mindestens fünf behelmte Syrinxbläser folgen mit ihren übergroßen Instrumenten innerhalb einer Prozession auf der Situla Welzelach (Taf. II: 4) drei Frauen nach, ${ }^{18}$ von M. Egg $(1990,22)$ als „Militärkapelle" angesprochen. Hingegen fallen auf der Situla Magdalenska gora-Preloge (Tumulus II/Grab a) die beiden Tanzenden zwischen den zwei behelmten Leierspielern durch ihre Kult-Kleidung auf. Dieser Springtanz ist die bis jetzt einzige Wiedergabe eines Tanzes innerhalb der Situlenkunst (Taf. IV: 5).

2. anhand des Hutes mit breiter Krempe:

Vor allem tragen diesen Hut die gemeinsam beim Fest Musizierenden auf den venetischen Situlen (Taf. I: 5; Eibner A. 2018a, Taf. 16: 31c-e) ebenso zum Teil die Ankommenden sowie die Zuhörer und Zu- schauer bei den Festspielen (Eibner A. 2018a, Taf. 1: 2; 8: 18, 20a; 10: 25a, d, e; 14: 29a-c).

\section{Musikerinnen}

Zur Stellung der Geschlechter - Musikerinnen sind nur im Osthallstattkreis zu finden. Im Bereich der Situlenkunst werden alle Instrumente von Männern gespielt. Nur im Osthallstattkreis finden sich Musikerinnen dargestellt, die auf einer Rahmenleier vom Typ Sopron spielen. So geht auf einem Fragment aus Sopron (Taf. IV: 1) eine Musikerin in weitem Glockenrock hinter einem Musiker in Hosen nach, während sich weitere Musikerinnen in Graphitmalerei noch im östlichen Bereich der Kalenderbergkultur finden (Taf. IV: 2; Eibner A. 2011, 29-34, Abb. 30-32; Gleirscher 2009, Abb. 7; Lochner 2011, 10-12, Abb. 5-8).

\section{DARSTELLUNGEN VON TÄNZEN}

Darstellungen von Tänzen sind aus beiden Bereichen bekannt. Im Osthallstattkreis finden sich Tänzer und Tänzerinnen: Auf einem Gefäß von Sopron/Tumulus 28 sind es u. E. vier Tanzpaare, drei in Hosen und ein Paar in weitem Glockenrock (Taf. I: 4), die sich alle am Bauch eingeritzt durch senkrechte Kannelurbündel voneinander getrennt unterhalb der am Hals dargestellten Leierspieler erkennen lassen. Auf dem Gefäß von Reichersdorf finden sich um den Leierspieler herum zwei Frauenpaare (Taf. IV: 3), während in Frög ebenfalls Tanzpaare aber in Basarabimanier dargestellt sind (Gleirscher 2009, Abb. 7). Im Osthallstattkreis scheinen daher Paartänzer - in Hosen oder Glockenrock - wiedergegeben zu sein.

Innerhalb der Situlenkunst zeigt die einzige Darstellung auf der Situla Magdalenska gora-Preloge (Tumulus II/Grab a) eine besondere Tanzform Springtänzer in einer Art Kultkleidung (Taf. IV: 5; Eibner A. 2012, 172 f., Taf. 4: 13).

Waffentänzer finden sich in Punkt-Buckel-Technik dargestellt und auf Felsbildern: In starker Bewegung sind die Waffentänzer auf der Rückenlehne des Biselliums (Taf. IV: 4) aus dem Fürstengrab von Hochdorf-Eberdingen wiedergegeben. Statisch hingegen lassen sie sich auf der Situla Typus Kurd von Sesto Calende/Kriegergrab B erkennen (Taf. IV: 6). Sie finden sich aber auch auf Felsbildern in der Valcamonica (Eibner A. 2008, 179-181, Taf. 10). Diese Darstellungen zeigen uns, dass die überlieferten

\footnotetext{
${ }^{18}$ Nach antiker Überlieferung werden Prozessionen von Musikern auch mit Tänzen begleitet (Tomedi 2001, 22 f.).
} 
Waffentänze der Salier mit ihren uralten Gesängen in kultischen Gewändern sogar außerhalb Latiums nachweisbar sind und auch noch in einigen Städten der Gallia Cisalpina sowie im norditalischen Gebiet verbreitet als Felsbilder (Eibner A. 2012, 177-180, 185 mit Anm. 99 u. 100, Taf. 6: 18-23) zu finden sind oder in Städten wie Mantua, die in den etruskischen (Einfluss-) Bereich gehörten. Wir hätten damit u. E. - in etwa zeitgleich - auch eine Bestätigung für außerrömische Waffentänze ähnlich den Saliern gegeben.

\section{ZUSAMMENFASSUNG}

Zunächst sind Musikinstrumente Bild überliefert und zwar aus allen bekannten Kategorien wie Chordophone, Aerophone, Membranophone und Idiophone, wobei im Osthallstattkreis und im Gebiet der Situlenkunst unterschiedliche Instrumentenformen vertreten sind: Als Saiteninstrumente sind Hallstattleiern und Harfen bzw. Situlenleiern verwendet worden, während sich bei den Blasinstrumenten einerseits der Hallstatt-Aulos, andererseits Syringen und Hörner finden. Ihre Musik diente zur musikalischen Umrahmung der Feste, zum Aufspielen beim Tanz und bei musischen Agonen. Hörner finden sich ausnahmslos als Signal gebende Instrumente in Händen vollgerüsteter Krieger. Diese sind als Anführer sowohl von Infanterie- als auch von Reitereinheiten dargestellt, aber auch als Einzelkrieger auf Votivblechen.

Originalfunde von Instrumenten zeigen uns aber, dass die Instrumente weiter verbreitet waren, als es die Bildquellen anzeigen: So sind Syringen aus Schafsknochen in Polen bzw. aus Holunderröhren in Deutschland bekannt. Die Carnyx findet sich von Frankreich bis zum Donauknie, also im Gebiet der Kelten verbreitet, so auch in Italien und England. Ein Leierspieler ist als Steinstatue in Frankreich erhalten geblieben.

Rasseln als Ideophone zeigen eine große Verbreitung und sind in verschiedenen Ausführungen auch im Original überliefert, während ein Tongefäß aus einem Gräberfeld in Niederösterreich als Membranophon, Trommel (?) anzusprechen ist. Aus den Darstellungen sind Paar-bzw. Reigentänze (?) sowie als besondere Tanzformen Spring- und Waffentänze zu erschließen. 

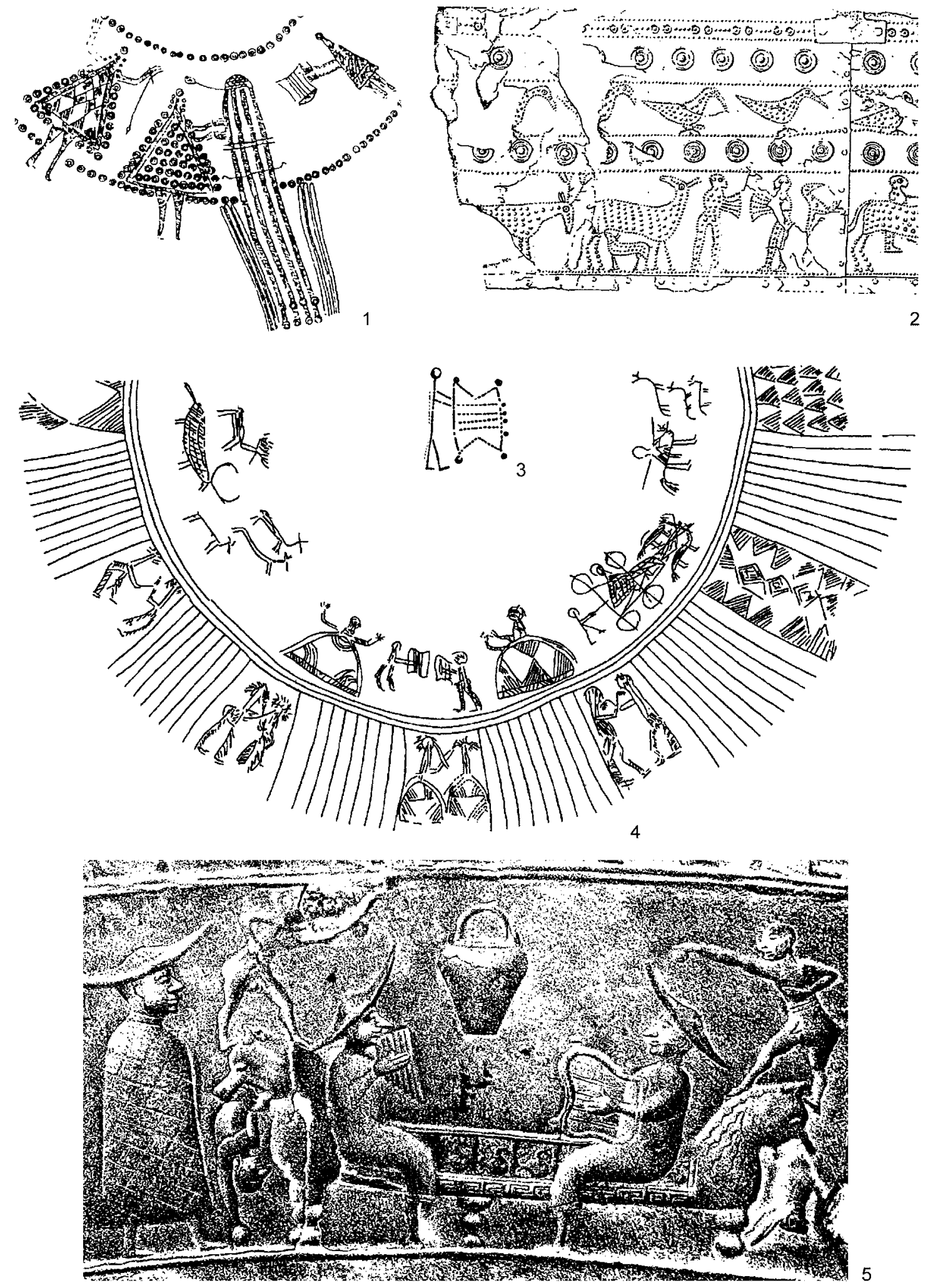

Taf. I. Leierinstrumente/Chordophone, verschiedene Leierformen. 1 - Hallstattleier: Sopron, Tumulus 27 (nach Eibner A. 2018a, Taf. 15: 30c); 2 - Hallstattleier: Sesto Calende, Kriegergrab A (nach Eibner A. 1981, Abb. 13); 3 - Rahmenleier vom Typ Oberpfalz: Schirndorf, Hügel 65 (nach Eibner A. 1994, Taf. 1: 1; 2: 5); 4 - Rahmenleier vom Typ Sopron: Sopron, Tumulus 28 (nach Eibner A. 2017, Abb. 6: 6); 5-Situlenleier: Bologna-Certosa, Situla Certosa (nach Eibner A. 2018, Taf. 20: 37c). 


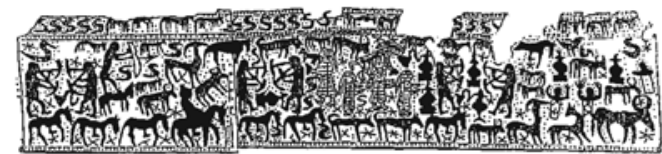

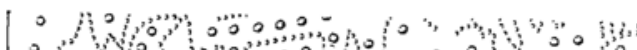

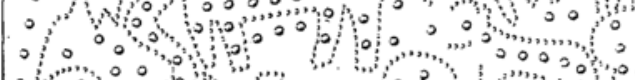

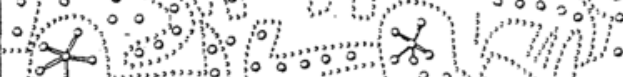

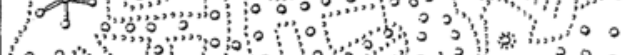

\%

0

0

0

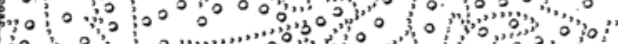

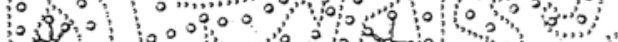

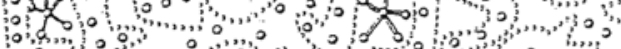

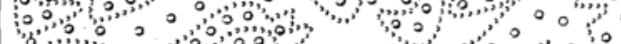

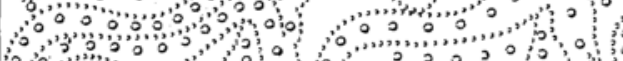

a o o o o

, 100 10, 010

a
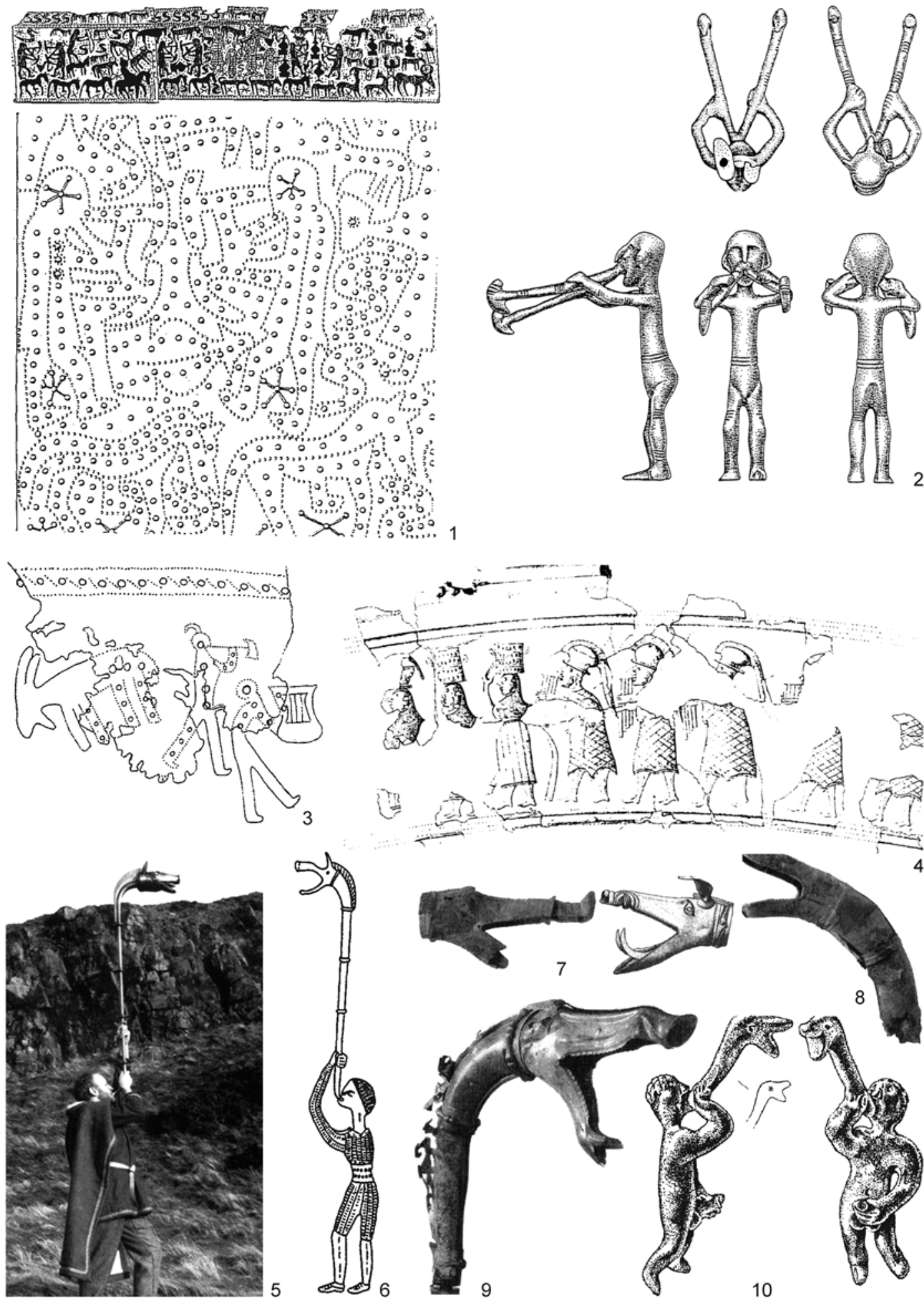

7
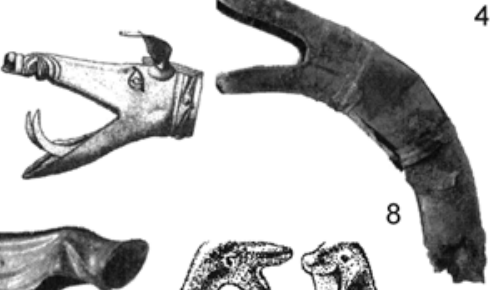


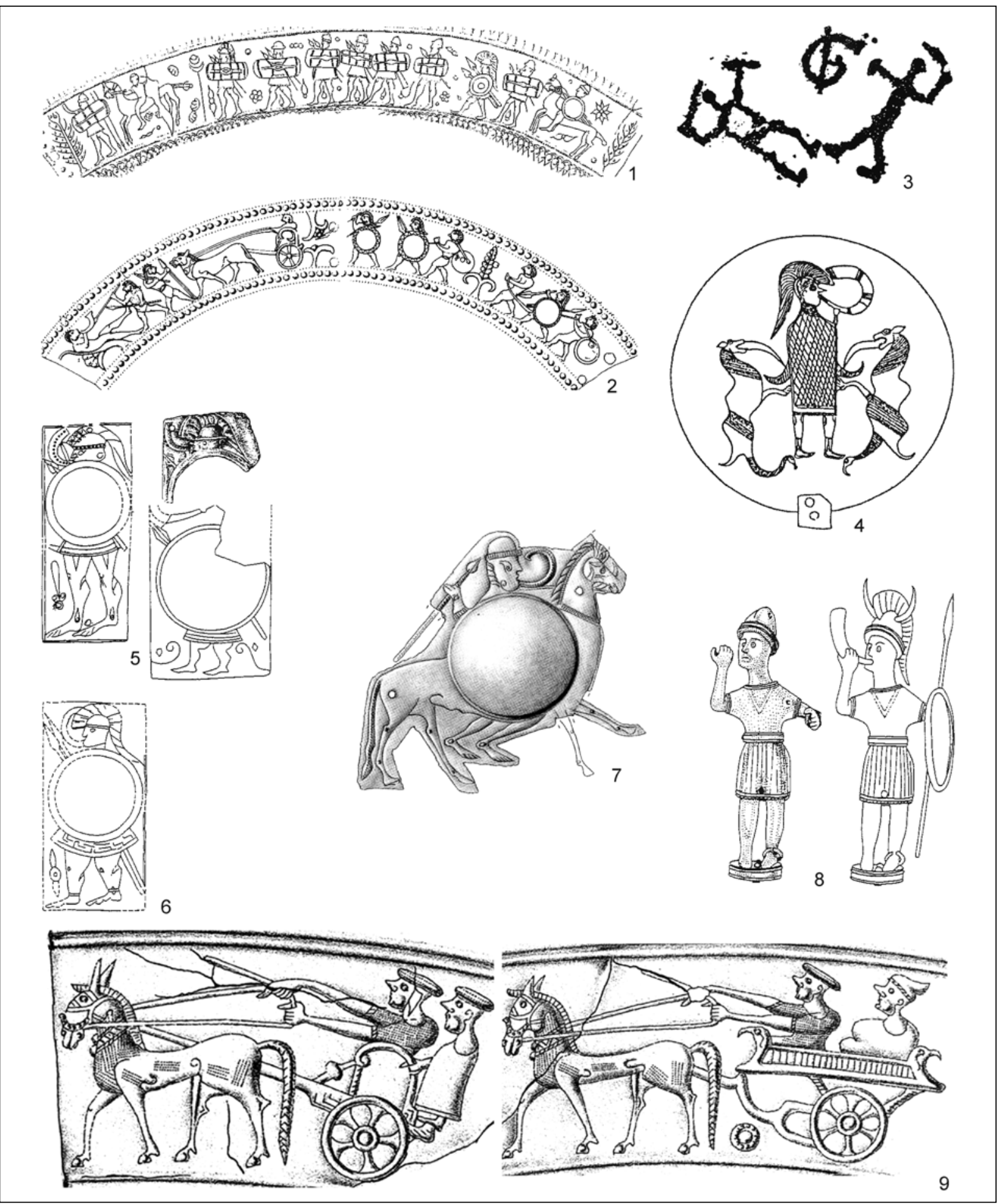

Taf. III. Hornbläser in kriegerischem Zusammenhang sowie Bronzeschellen am Halsgurt des Pferdegeschirrs. 1 - Bologna-Arnoaldi: Situla Arnoaldi, Anführer eines Kriegerzuges von Infanteristen; 2 - Este-Villa Benvenuti: Situla Este-Benvenuti, Bigafahrer eilt Hornbläser zu Hilfe; 3 - Felsbild aus der Valcamonica-Bedolina, Fels 16: Horn mit Hornfessel zwischen Kriegern; 4 - Bologna-Arnoaldi: Spiegel Arnoaldi, eingraviertes behelmtes Numen; 5, 6 - estensische Heiligtümer in Baratela (5) und Caldevigo (6): Votivbleche mit Hornbläsern, gerüstet mit Helm, Schild und Lanze; 7 - Este-Reitia Heiligtum: Reiterkriegerblech als Votiv mit zwei Löchern; 8 - Idrija pri Bači: Bronzestatue, rekonstruiert mit Horn, Schild und Lanze sowie mit Helmzimier und -busch; 9 - Vače-Nad Lazom: Situla Vače, je zwei Bronzeschellen am Halsgurt von Pferden (1-8 nach Eibner A. 2018b, Abb. 5: a; 7: a-f; 9 nach Eibner A. 2018a, Abb. 3: a; 4: 8d, 8h). 

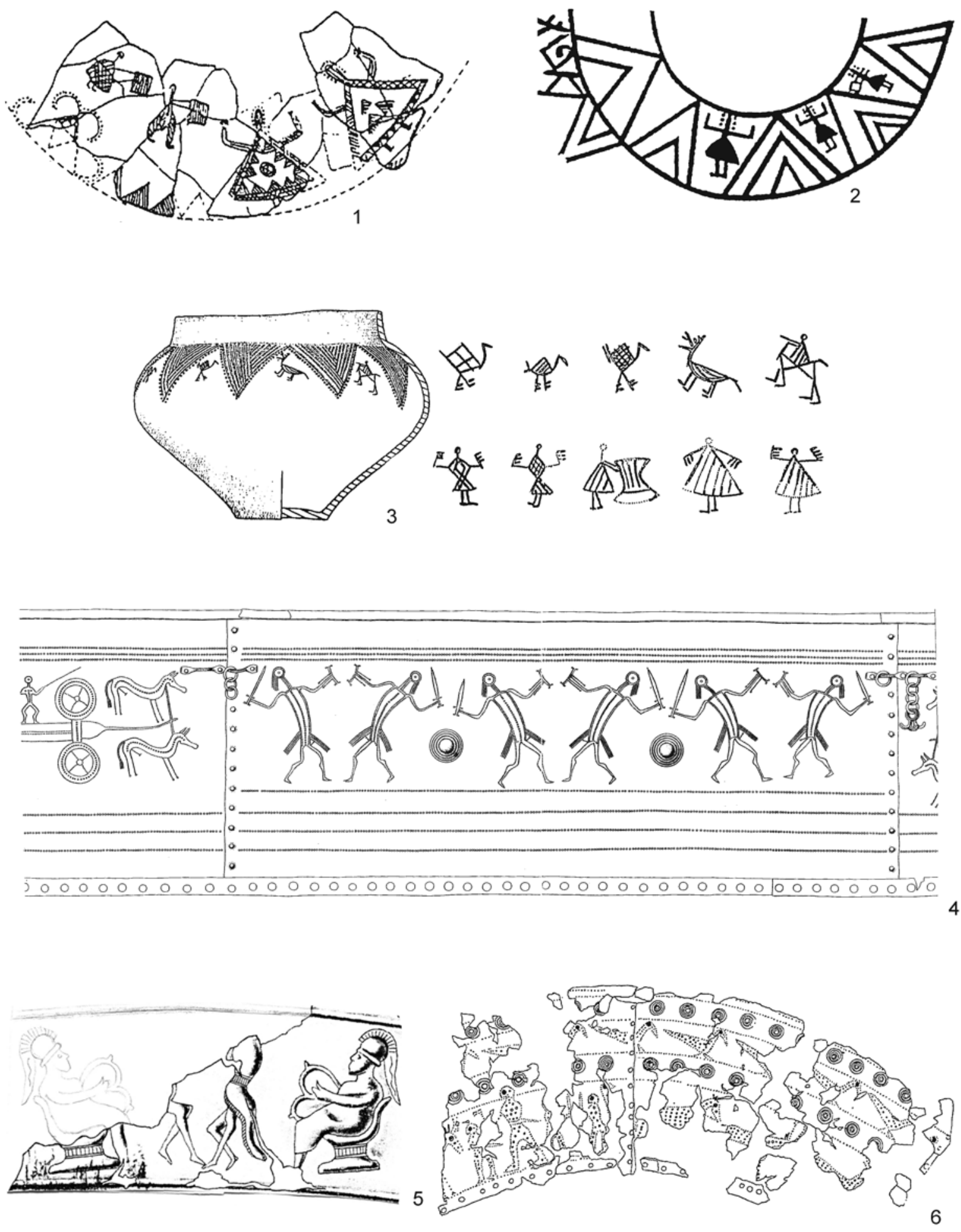

Taf. IV. Musikerinnen mit Rahmenleier vom Typ Sopron, verschiedene Tanzformen. 1 - Musikerin mit Rahmenleier: Sopron, Unbekannter Tumulus - Tumulus 96 oder 98 (nach Eibner A. 1994, Taf. 1: 1; 2: 1); 2 - Musikerin mit Rahmenleier: Dolné Janíky, Tumulus II (nach Eibner A. 2017, Abb. 6: a); 3 - Musiker mit Hallstattleier spielt zum Tanz auf: Reichersdorf, Grab 20 (nach Eibner A. 2017, Abb. 6: c); 4 - Waffentänzer: Kline Hochdorf, drei Paare mit Schwertern, in stark bewegtem Tanz (nach Eibner A. 2018a, Taf. 20: 38b); 5 - Springtanz, zwei behelmte Leierspieler spielen für die Tänzer auf: Situla Magdalenska gora, Tumulus II/Grab a (nach Eibner A. 2018a, Taf. 20: 38a); 6 - Waffentänzer: Sesto Calende, Kriegergrab B, statische Tanzwiedergabe (nach Eibner A. 1981, Abb. 14). 


\section{LITERATUR}

Dobiat 1982 - C. Dobiat: Menschendarstellungen auf ostalpiner Hallstattkeramik. Eine Bestandsaufnahme. Acta Archaeologica Academiae Scientiarum Hungaricae 34, 1982, 279-322.

Egg 1990 - M. Egg: Urgeschichtliche Bronzehelme aus dem schweizerischen Alpenraum. Helme vom „Negauer" Typ. Helvetia Archaeologica 81, 1990, 2-27.

Egg 2012 - M. Egg: Die eisenzeitlichen Waffenweihungen in den Ostalpen. In: W. Meighörner (Hrsg.): Waffen für die Götter. Krieger - Trophäen - Heiligtümer. Ausstellung im Tiroler Landesmuseum Ferdinandeum vom 7. 12. 2012 - 31. 3. 2013. Innsbruck 2012, 100-108.

Egg 2013 - M. Egg: Zur figuralen Kunst von Kleinklein. In: M. Egg/D. Kramer (Hrsg.): Die hallstattzeitlichen Fürstengräber von Kleinklein in der Steiermark: der Kröllkogel. Monographien des Römisch-Germanischen Zentralmuseums Mainz 110. Mainz 2013, 447-472.

Egg/Munir 2013 - M. Egg/J. Munir: Metallfunde. In: M. Egg/ D. Kramer (Hrsg.): Die hallstattzeitlichen Fürstengräber von Kleinklein in der Steiermark: der Kröllkogel. Monographien des Römisch-Germanischen Zentralmuseums Mainz 110. Mainz 2013, 75-279.

Eibner A. 1981 - A. Eibner: Darstellungsinhalte in der Kunst der Hallstattkultur. Gedanken zum ,"überhöhten Leben" im Situlenbereich und Osthallstattkreis. In: C. Eibner (Hrsg.): Die Hallstattkultur. Bericht über das Symposium in Steyr 1980 aus Anlaß der Internationalen Ausstellung des Landes Oberösterreich. Linz 1981, 261-296.

Eibner A. 1986 - A. Eibner: Musikleben in der Hallstattzeit. Betrachtungen zur „mousiké“ anhand der bildlichen Darstellungen. In: M. von Albrecht/W. Schubert (Hrsg.): Musik in Antike und Neuzeit. Quellen und Studien zur Musikgeschichte von der Antike bis zur Gegenwart 1. Frankfurt 1986, 271-320.

Eibner A. 1994 - A. Eibner: Music during the Hallstatt period. Observations on Mousiké as depicted on Iron Age circumalpine vessels. In: C. Homo-Lechner/ A. Bélis (éds.): La pluridisciplinarité en archéologie musicale: colloque organisé en hommage à Théodore Reinach. Vol. 1. $\mathrm{IV}^{\mathrm{e}}$ recontres internationales d'archéologie musicale de l'ICTM Saint-Germain-en-Laye, 8-12 octobre 1990. Paris 1994, 299-320.

Eibner A. 1999 - A. Eibner: Der Aulosbläser von Százhalombatta und sein kulturgeschichtliches Umfeld. Versuch einer Interpretation. In: E. Jerem/I. Poroszlai (eds.): Archaeology of the Bronze and Iron Age. Experimental Archaeology-Environmental - Archaeological Parks. Proceedings of the International Archaeological Conference Százhalombatta, 3-7 October 1996. Archaeolingua 9. Budapest 1999, 37-52.

Eibner A. 2000 - A. Eibner: Die Rolle des Hornbläsers in der eisenzeitlichen Kampftechnik anhand der Situlendenkmäler. Ein Beitrag zum musikarchäologischen Kulturkontakt. In: E. Hickmann/I. Laufs/R. Eichmann (Hrsg.): Studien zur Musikarchäologie II. Musikarchäologie früher Metallzeiten. Vorträge des 1. Symposiums der International Study Group on Music Archaeology im Kloster Michaelstein, 18.-24. Mai 1998. Orient-Archäologie 7. Rahden/Westf. 2000, 313-324.
Eibner A. 2008 - A. Eibner: Der Faustkampf - ein Aufnahmeritus in den Kriegerstand? Gedanken zu einem integrierenden Bestandteil des „,Situlenfestes“. In: B. Gediga/ W.Piotrowski(Hrsg.): Urgeschichtliche und frühmittelalterliche Kunst als historische Quelle. Biskupiner Archäologische Arbeiten 6. Biskupin - Wrocław 2008, 151-195/198.

Eibner A. 2011 - A. Eibner: 2. Musikausübung in der Hallstatt-Kultur. 2.1 Komposition, Aufführung, Musizierpraxis. In: M. Lochner (Hrsg.): Sitularia - Klänge aus der Hallstattzeit (gespielt auf rekonstruierten antiken Instrumenten). Mitteilungen der Prähistorischen Kommission der Österreichischen Akademie der Wissenschaften 76. Wien 2011, 29-39.

Eibner A. 2012 - A. Eibner: Männer in Waffen: Kämpfer oder Tänzer? In: P. Anreiter/E. Bánffy/L. Bartosiewicz/ W. Meid/C. Metzner-Nebelsick (eds.): Archaeological, Cultural and Linguistic Heritage. Festschrift for Erzsébet Jerem in Honour of her $70^{\text {th }}$ Birthday. Archaeolingua 25. Budapest 2012, 159-194.

Eibner A. 2013 - A. Eibner: Zu den Schalltrichtern eisenzeitlicher Blechblasinstrumente. Vom Horn zur Karnyx. In: R. Breitwieser/M. Frass/G. Nightingale (Hrsg.): Calamus. Festschrift für Herbert Graßl zum 65. Geburtstag. Philippika. Altertumskundliche Abhandlungen 57. Wiesbaden 2013, 157-176.

Eibner A. 2017 - A. Eibner: Kultwagen mit Figuren aus Keramik im Osthallstattkreis? Studia Archaeologica Brunensia 22/1, 2017, 71-90.

Eibner A. 2018a - A. Eibner: Motiv und Symbol als Ausdrucksmittel der Bildsprache in der eisenzeitlichen Kunst. Przeglad Archeologiczny 66, 2018, 77-136.

Eibner A. 2018b-A. Eibner: Darstellungen von Kriegern und Kampfgeschehen in der Situlenkunst. Mitteilungen der Anthropologischen Gesellschaft in Wien 148, 2018, 101-119.

Eibner C. 2012 - C. Eibner: Hallstättische Sagen und Mythen. In: P. Anreiter/E. Bánffy/L. Bartosiewicz/ W. Meid/C. Metzner-Nebelsick (eds.): Archaeological, Cultural and Linguistic Heritage. Festschrift for Erzsébet Jerem in Honour of her $70^{\text {th }}$ Birthday. Archaeolingua 25. Budapest 2012, 195-205.

Erker 2018 - M. Erker: Musik, Tanz und Krieg - eine antike Kombination? Mitteilungen der Anthropologischen Gesellschaft in Wien 148, 2018, 121-135.

Gleirscher 2009 - P. Gleirscher: Sopron - Nové Košariská Frög. Zu den Bildgeschichten der Kalenderbergkultur. Prähistorische Zeitschrift 84, 2009, 202-223.

Gleirscher 2014 - P. Gleirscher: 13. Fragments of a carnyx from Leisach (Austria). In: Chr. Gosdon/S. Crawford/ K. Ulmschneider (eds.): An offprint from celtic art in Europe making connections. Essays in honour of Vincent Megaw on his $80^{\text {th }}$ birthday. Oxford (Philadelphia) 2014, 113-118.

Haas 1985 - G. Haas: Die Syrinx in der griechischen Bildkunst. Wiener Beiträge 11. Wien - Köln - Graz 1985.

Hornbostel/Sachs 1914 - E. M. Hornbostel/C. Sachs: Systematik der Musikinstrumente. Ein Versuch. Zeitschrift für Ethnologie 46, 1914, 553-590.

Hunter 2019 - F. Hunter: The carnyx of Iron Age Europe: the Deskford carnyx in European context. Monographien des Römisch-Germanischen Zentralmuseums Mainz 146. Mainz 2019. 
Krausse u. a. 2017 - D. Krausse/N. Ebinger-Rist/S. Million/ A. Billambez/J. Wahl/E. Stephan: The 'Keltenblock' project: discovery and excavation of a rich Hallstatt grave at the Heuneburg, Germany. Antiquity 91, 2017, 108-123.

Laharnar/Turk 2018 - B. Laharnar/P. Turk: Iron Age stories from the crossroads. Ljubljana 2018.

Lobisser, im Druck - W. Lobisser: Die Kleinfunde aus Holz aus der eisenzeitlichen Gewerbesiedlung im Ramsautal am Dürrnberg bei Hallein. Dürrnberg-Forschungen 13: Abteilung Siedlung. Bochum, im Druck.

Lochner 2011 - M. Lochner: 1. Zur Musikarchäologie der Hallstattkultur. In: M. Lochner (Hrsg.): Sitularia Klänge aus der Hallstattzeit (gespielt auf rekonstruierten antiken Instrumenten). Mitteilungen der Prähistorischen Kommission der Österreichischen Akademie der Wissenschaften 76. Wien 2011, 7-28.

Pöll/Tomedi/Zanier 2019 - J. Pöll/G. Tomedi/W. Zanier: Funde der späten Eisen- und frühen römischen Kaiserzeit vom Pircherwald in Oberhofen (Tirol). In: W. Zanier (Hrsg.): Kulturwandel um Christi Geburt. Spätlatène- und römische Kaiserzeit in den mittleren Alpen zwischen Südbayern und Gardasee. Akten des Kolloquiums in Innsbruck am 18. und 19. Oktober 2017. Münchner Beiträge zur Vor- und Frühgeschichte 67/1-2. München 2019, 93-143.

Pomberger 2016 - B. M. Pomberger: The Development of Musical Instruments and Sound Objects from the Late Bronze Age to the La Tène Period in the Area between the River Salzach and the Danube Bend. In: B. Gediga/A. Grossman/W. Piotrowski (Hrsg.): Europa zwischen 8 Jhd. v. Chr. Geb. bis 1 Jhd. u. Zeit. Biskupiner Archäologische Arbeiten 11. Biskupin - Wrocław 2016, 45-74/76.

Manuskript angenommen am 26. 7. 2020

Abstract translated by Barbara Eibner

Súhrn preložila Anita Kozubová

Prof. Dr. Alexandrine Eibner

Alszeile 118/10/4

A - 1170 Wien

alexandrine.eibner@gmx.at
Pomberger 2017 - B. M. Pomberger: Bronzebommeln und Schellen - eine klangliche Errungenschaft aus der Spätbronze- und frühen Eisenzeit. Studia Archaeologica Brunensia 22/2, 2017, 15-34.

Préhistoire 2002 - Préhistoire: Préhistoire de la musique. Sons et instruments de musique des âges du Bronze et du Fer en France. Exposition du 17 mars au 10 novembre 2002 au Musée de Préhistoire de Nemours. Nemours 2002.

Rieckhoff 2002 -S. Rieckhoff: Die Religion der Kelten. In: H.-U. Cain/S. Rieckhoff (Hrsg.): fromm - fremd - barbarisch. Die Religion der Kelten. Eine Sonderausstellung der Universität Leipzig 13. 4. 2002 - 15. 6. 2002. Mainz 2002, 128-179.

Salzani 1976 - L. Salzani: La fine dell'età del Bronzo e la prima età dell Ferro nel territorio veronese. In: A. Aspes (ed.): 3000 anni fa a Verona dalla fine dell'età del Bronzo all'arrivo dei Romani nel territorio veronese. Catalogo della mostra omonima, allestita a Verona dal 1 luglio al 31 dicembre 1976. Verona 1976, 29-62.

Sölder 2008 - W. Sölder: Das Fragment einer latènezeitlichen Panflöte aus Sanzeno, Trentino. In: C. Sporer-Heis (Hrsg.): Tirol in seinen alten Grenzen. Festschrift für Meinrad Pizzinini zum 65. Geburtstag. Schlern-Schriften 341. Innsbruck 2008, 223-245.

Tomedi 2001 - G. Tomedi: Zur vorgeschichtlichen Musik in Alttirol und im Südalpenraum. In: K. Drexel/M. Fink (Hrsg.): Musikgeschichte Tirols. Band 1: Von den Anfüngen bis zur Frühen Neuzeit. Schlern-Schriften 315. Innsbruck 2001, 11-35.

Veres 2009-J. Veres: The depiction of a carnyx-player from the Carpathian Basin - a study of two Celtic bronze statuetes from Eastern Hungary. Archäologisches Korrespondenzblatt 39, 2009, 231-249.

Prof. Dr. Clemens Eibner

Alszeile 118/10/4

A - 1170 Wien

clemens.eibner@zaw.uni-heidelberg.de 


\title{
Hudobné nástroje a hudobná prax $\mathrm{v}$ dobe železnej $\mathrm{v}$ strednej Európe - na základe ikonografických prameňov a archeologických nálezov
}

\author{
Alexandrine Eibner - Clemens Eibner
}

\begin{abstract}
SÚHRN
Príspevok je venovaný strunovým a dychovým nástrojom známym vo východohalštatskej kultúre a v situlovom umení, ako aj lýram zo západohalštatskej kultúry. Primárne pramene $\mathrm{k}$ hudobným nástrojom zo sledovaného priestoru a obdobia predstavujú ikonografické pramene - vo východohalštatskej kultúre a v situlovom umení nachádzame ich všetky známe kategórie, ako sú strunové nástroje, aerofóny, membranofóny či idiofóny. Zo strunových nástrojov sa používali lýry halštatského typu, doskové (rámové) citary šopronského typu a harfy, resp. lýry tzv. situlového typy, zatial' čo z dychových nástrojov sú doložené syringy, trubky a aulosy halštatského typu. Melódie vydávané z týchto hudobných nástrojov boli

súčastou rôznych osláv a tiež tanečných a hudobných sútaží. Trubky ako dychové nástroje boli často zobrazované na votívnych plaketách v rukách bojovníkov v plnej výzbroji. Ikonografické pramene umožňujú identifikovał rôzne kategórie spevákov a tanečníkov ako interpretov, sólistov a súborov/skupín. Výrobky situlového umenia zároveň poskytujú indície o sociálnom statuse spevákov a tanečníkov a vo východohalštatskej kultúre sú vyobrazenia tanečníc a speváčok dôležitým prameňom pri riešení problematiky postavenia žien $\mathrm{v}$ miestnych spoločenstvách. V prípade tanca boli zobrazované párové a kruhové tance a tiež zvláštne formy skokových tancov a tancov so zbraňami.
\end{abstract}


